\title{
Zur Mobilisierung von politischen Beteiligungsrechten für nicht EU-angehörige Einwanderer*
}

\author{
Klaus Sieveking
}

Die Mobilisierungsdebatte wurde in der Rechtssoziologie in Deutschland erstmals in den 1980er Jahren geführt. ${ }^{1}$ Anfang der 90er Jahre wurden die rechtssoziologischen Anstöße zu einer rechtsmethodischen Debatte erneut aufgegriffen um einen Zusammenhang zwischen wichtigen gesellschaftspolitischen Themen und der Fortentwicklung juristischer Methoden zur Durchsetzung politischer Zielsetzungen herzustellen. Diese Fragestellungen fanden unter dem Einfluss der mit der Vereinigung Deutschlands neu entstandenen politischen und gesellschaftlichen Probleme besonderes Interesse. Man versuchte, das Verhältnis von Rechtsdogmatik und juristischer Praxis neu zu thematisieren. ${ }^{2}$ Eine nachhaltige Wirkung haben diese Debatten nicht gehabt. So ist die Anwendung dieser rechtssoziologischen Methode bislang weitgehend ausgeblieben. Dagegen fanden Methoden empirischer Sozialforschung vor allem Eingang in Untersuchungen zu Verfahren und Struktur von gerichtlichen Streitigkeiten oder in Berichte aus der Ministerialbürokratie zur Gesetzesvorbereitung.

\section{Zur Bedeutung der Frage nach der Mobilisierung von Recht}

Das vorliegende Thema der Mobilisierung von politischen Beteiligungsrechten von Migranten in Deutschland lenkt den Blick auf den Zusammenhang von politischer Zielsetzung und den Formen ihrer Durchsetzung seitens interessierter Akteure. Eng damit verbunden ist ein Ausschnitt aus der aktuellen Debatte über die Integration der auf Dauer in Deutschland lebenden Einwanderer. ${ }^{3}$ Dabei geht es um Wege und Mittel der Integration von Immigranten in die Aufnahmegesellschaft einerseits und um die Aufrechterhaltung und Weiterentwicklung des gesellschaftlichen Zusammenhalts andererseits, also das Wie des

* Dem Beitrag liegt ein englischsprachiges Manuskript mit dem Titel „Introduction of Political Participation Rights for Non-EU-National Immigrants in Germany" für den von der griechischen Stiftung für Europäische und Auswärtige Politik (ELIAMEP) im Namen der European Science Foundation veranstalteten Workshop „Rights, Legal Mobilization and Political Participation in Europe" vom 8. bis 10. Oktober 2009 in Athen zugrunde.

1 Vgl. Erhard Blankenburg, Mobilisierung von Recht, in: Zeitschrift für Rechtssoziologie, 1. Jg. (1980), H. 1, S. $33-64$.

2 Siehe ders., Mobilisierung des Rechts: eine Einführung in die Rechtssoziologie, Berlin 1995. Siehe auch die jüngst erneuerte Debatte bei: Gesine Fuchs / Sandra Konstatzky / Doris Liebscher / Sabine Berghahn, Rechtsmobilisierung für Lohngleichheit, in: Kritische Justiz 2009, S. 253 270. Mit einem Ländervergleich Deutschland, Österreich und der Schweiz, die rechtliche (,legal opportunity structure"), gesellschaftliche und individuelle Einflussfaktoren der Rechtsmobilisierung unterscheiden.

3 Siehe Axel Schulte, Politikkonzepte für eine multikulturelle Einwanderungsgesellschaft, in: Einwanderungsgesellschaft Deutschland. Wege zu einer sozialen und gerechten Zukunft, Tagungsdokumentation des Gesprächskreises Migration und Integration der Friedrich-Ebert-Stiftung, WISO-Diskurs, Berlin 2009, S. 17 - 44, S. 32 ff. mit zahlreichen Hinweisen auf einschlägige Literatur. 
Zusammenlebens von Einheimischen und Eingewanderten. Unmittelbarer Bezugspunkt ist die Frage nach den Bedingungen und Formen politischer Partizipation im Sinne einer „Integration durch Partizipation". Ob Beteiligung in Form des Kommunalwahlrechts oder Einbürgerung hierfür das geeignete Instrument ist, wird kontrovers diskutiert. ${ }^{4}$

Exemplarisch soll hier die historische Entwicklung verfolgt werden, wie gesellschaftspolitische Forderungen nach politischer Beteiligung in rechtlich verbindliche Strukturen umgesetzt wurden, unter welchen Bedingungen dies geschieht und welche Aspekte hierbei hinderlich sind. Angesichts unterschiedlicher Phasen dieser Interessenartikulierungen lassen sich vorläufige Schlussfolgerungen für das spezifische Verhältnis von Gleichbehandlungsinteressen der Zuwanderer und Ängsten der Aufnahmegesellschaft in Deutschland formulieren.

\section{Zur Situation der Migranten in Deutschland}

Deutschland hatte 2009 (Stand: 31. August) 81.842.400 Einwohner. Die ausländische Bevölkerung betrug 7.146.600 (8,7 Prozent), im Ausländerzentralregister waren Ende 2009 rund 6,69 Millionen Ausländer registriert. Von diesen hatten über 2,3 Millionen (35,3 Prozent) die Staatsangehörigkeit eines Mitgliedstaates der Europäischen Union5, 4.332.134 (64,7 Prozent) sind Drittstaatsangehörige.

Von dem Ausländerbestand nach dem Ausländerzentralregister am 31. Dezember 2009 in Höhe von 6.694.766 Personen kommen beispielsweise 1.658.03 (24,8 Prozent) aus der Türkei und 221.222 aus Kroatien.

Der größte Teil der nichtdeutschen Bevölkerung besteht aus Personen, die im Zuge der Anwerbung von ausländischen Arbeitskräften und des Familiennachzuges nach Deutschland gekommen sind oder als deren Kinder bereits hier geboren sind. Ende 2008 lebten circa 2,4 Millionen Ausländer schon länger als 20 Jahre in Deutschland, weitere 950.000 mehr als zehn Jahre. Die durchschnittliche Aufenthaltsdauer der Ausländer in Deutschland betrug im Jahre 2009 18,6 Jahre. Mehr als zwei Drittel der ausländischen Kinder und Jugendlichen sind hier geboren. ${ }^{6}$

Die ausländische Bevölkerung verteilt sich nicht gleichmäßig: Rund neun Zehntel leben in den alten Bundesländern, acht Zehntel in westdeutschen Großstädten. Der Anteil von ausländischen Einwanderern liegt in vielen größeren (west-)deutschen Städten heute bereits zwischen 20 und 30 Prozent. Das Statistische Landesamt Nordrhein-Westfalen hat berechnet, dass der Ausländeranteil in einigen Großstädten des Landes (ohne Berücksichtigung von Staatsbürgerschaftswechseln) bis Ende 2010 auf 40 Prozent und mehr steigen wird.

4 Näheres hierzu weiter unten bei 6.

5 Davon kommen 1.618.083 (24,2 Prozent) aus den 14 alten EU-Staaten, 577.25 (8,6 Prozent) aus den zehn seit 1. Mai 2004 und 166.834 (2,5 Prozent) aus den zwei seit 1. Januar 2007 neuen EU-Staaten.

6 Die hier angegebenen Zahlen sind der jüngsten Publikation „Ausländerzahlen 2009“ des Bundesamtes für Migration und Flüchtlinge vom Juli 2010 entnommen. Weitere Angaben und Differenzierungen zum Beispiel nach der Bevölkerung mit Migrationshintergrund sind ausführlich dokumentiert in dem Migrationsbericht des Bundesamtes für Migration und Flüchtlinge im Auftrag der Bundesregierung. Vgl. dies., Migrationsbericht 2008, Berlin 2010, S. 198 ff. und Tabellenanhang, S. $199 \mathrm{ff}$. 
Auch innerhalb der Städte verteilen sich die Einwanderer nicht gleichmäßig. Sie konzentrieren sich in benachteiligten Stadtteilen. Hier hat sich die Schere zwischen Wahlbevölkerung und Wohnbevölkerung besonders weit geöffnet. Daraus wird deutlich, wie wichtig die aktive politische Beteiligung von Ausländern gerade auf der kommunalen Ebene innerhalb der dafür vorgesehenen politischen Gremien ist. ${ }^{7}$

\section{Zum Begriff der politischen Beteiligung}

Seitdem zu Beginn der 1970er Jahre über die politische Beteiligung von Migranten an integrationsrelevanten gesellschaftlichen Entscheidungsprozessen in der Bundesrepublik Deutschland nachgedacht worden ist, bezogen sich konkrete Vorschläge hierzu entweder auf die Einräumung von Wahlrechten oder auf andere Mitwirkungsmöglichkeiten auf kommunaler Ebene. Das Leben in der Kommune führte im Laufe der Zeit zur Herausbildung vielfältiger Instrumente politischer Mitwirkung: Im derzeitigen „Nationalen Integrationsplan“ stehen im „Handlungsfeld gesellschaftliche Integration“ heute insbesondere Mitgestaltungsformen im Blick, die sowohl Migranten ${ }^{8}$, Unionsbürgern und Drittstaatsangehörigen offen stehen. Dies sind beispielsweise Ratsausschüsse als Unterorgane der Gemeindevertretungen, Sachverständigen-Arbeitskreise, Jugendparlamente, Engagement im Quartiersmanagement und weitere Möglichkeiten des bürgerschaftlichen Engagements in Parteien, Kirchen und Religionsgemeinschaften, Gewerkschaften, Verbänden und Vereinen. Der Fokus der Aktivitäten im bundesweiten Integrationsprogramm liegt daher vor allem im Bereich „kommunaler Mitgestaltungsmöglichkeiten“?.

7 Vgl. Klaus Peter Strohmeier, Demographischer Wandel und soziale Segregation, in: Bertelsmann Stiftung (Hrsg.), Demographie konkret - Soziale Segregation in deutschen Großstädten, Gütersloh 2008, S. $10-14$.

8 In Deutschland lebten 2007 rund 15,4 Millionen Personen mit Migrationshintergrund; vgl. Bundesamt für Migration und Flüchtlinge, a.a.O. (Fn. 6), S. 211 ff. Nach der neueren Definition des Statistischen Bundesamtes spricht man von Personen mit Migrationshintergrund, wenn sie nicht auf dem Gebiet der heutigen Bundesrepublik geboren wurden und 1950 oder später zugewandert sind, keine deutsche Staatsangehörigkeit besitzen oder eingebürgert wurden. Deutsche haben auch dann einen Migrationshintergrund, wenn ein Elternteil mindestens eine der genannten Voraussetzungen erfüllt; vgl. Beauftragte der Bundesregierung für Migration, Flüchtlinge und Integration (Hrsg.), 7. Bericht über die Lage der Ausländerinnen und Ausländer in Deutschland, Berlin 2007, S. 12. Siehe auch Tanja Merkle, Lebenswelten in Deutschland, Ergebnisse aktueller Studien von Sinus Sociovision, in: Dirk Lange / Ayca Polat (Hrsg.), Unsere Wirklichkeit ist anders. Migration und Alltag, Bonn 2009, S. 62 - 79.

$9 \mathrm{Zu}$ neueren Entwicklungen der Ausländerbeiräte siehe das „Landesgesetz über die Einrichtung von kommunalen Beiräten für Migration und Integration" (Gesetz- und Verordnungsblatt Rheinland-Pfalz 2008, S. 294), das am 1. Januar 2009 in Kraft trat, vgl. Florian Edinger, Neue Beiräte als Motoren der Integration, in: Treffpunkt - Magazin für Migration und Integration, Heft 3/2008-1/2009, S. 3 - 6. In Berlin wird derzeit kontrovers über den Entwurf eines Gesetzes zur Regelung von Partizipation und Integration debattiert, dessen Notwendigkeit sich nach Ansicht der Senatorin Bluhm aus der demographischen Entwicklung ergibt und der klarstellen soll, dass ,interkulturelle Kompetenzen bei den Beschäftigten im Öffentlichen Dienst einen höheren Stellenwert bekommen und dass Menschen mit Migrationshintergrund (MMH) in Ämtern, Kitas und Schulen präsent sind.", vgl. In der Diskussion: Das Berliner Partizipations- und Integrationsgesetz, in: Berlin International, Nr. 71, Heft 7-8/2010, S. 4 - 10, S. 4. 
Es fehlt demgegenüber in diesem Programm an Handlungsvorschlägen, Expertisen, Initiativen und Modellprojekten, die explizit auf Formen der „politischen Partizipation“ ausgerichtet sind. Darunter wird im Folgenden das subjektive Recht auf Beteiligung an den Wahlen zu den Kommunalparlamenten verstanden, dessen Voraussetzungen im Grundgesetz, in den Landes- und Kommunalverfassungen sowie den Wahlgesetzen geregelt sind. Gemeint sind das aktive Wahlrecht, das heißt die Berechtigung, durch Stimmabgabe an der Wahl teilzunehmen, und das passive Wahlrecht, also gewählt werden zu dürfen. ${ }^{10}$ Beide Rechte sind an die deutsche Staatsangehörigkeit, den Wohnsitz in Deutschland und ein bestimmtes Alter (in der Regel 18 Jahre) gebunden. Lediglich Unionsbürger dürfen sich an Kommunalwahlen unter den gleichen Voraussetzungen wie Deutsche beteiligen.

Ganz anderen Aspekten widmet sich beispielsweise die Anfang 2009 erschienene Studie der Bertelsmann Stiftung „Demokratie und Integration in Deutschland“. ${ }^{11}$ Darin werden Fragen der politischen Führung und Partizipation aus Sicht von Menschen mit und ohne Migrationshintergrund erörtert. Die beteiligten Forscher interessieren sich zum Beispiel dafür wie Menschen in Deutschland die Demokratie und demokratische Politik sehen, wie ausgeprägt die demokratischen Haltungen bei Menschen mit und ohne Migrationshintergrund sind oder wie stark sie sich für Politik interessieren und engagieren. Die im Einzelnen vorgestellten Ergebnisse sind bemerkenswert, tragen allerdings zu dem hier im Vordergrund stehenden Thema keine Erkenntnisse bei, zumal man bei den befragten integrierten Menschen mit Migrationshintergrund die Eingebürgerten im Blick hatte, ihre bestehende Wahlberechtigung also unterstellt werden kann.

\section{Gesellschaftliche Debatten zwischen 1971 und 1989}

Es waren die für die „Sozialberatung der Ausländer“ zuständigen Spitzenverbände der Wohlfahrtsverbände, die erstmals die Forderung nach Gewährung des aktiven und passiven Kommunalwahlrechts erhoben. Bereits 1971, wenige Jahre nach Abschluss der Vereinbarungen zur Anwerbung ausländischer Arbeitnehmer, die damals noch Gastarbeiter genannt wurden, fasste der Zentralverband des Deutschen Caritasverbandes einen entsprechenden Beschluss. Die Bundeskonferenz des Verbandes der Arbeiterwohlfahrt (AWO) schloss sich den Forderungen an.

Der erste Ausländerbeauftragte der Bundesregierung, Heinz Kühn, behandelte das Thema in einem Memorandum von 1979, das unter anderem eine Übersicht der Befürworter enthält, zum Beispiel die Caritas, die Arbeiterwohlfahrt, die Gewerkschaften, den Deut-

10 Nicht behandelt werden hier also Beteiligungsrechte zum Beispiel im Rahmen von Betriebsratswahlen oder der Wahlen zu den Vertretungsorganen der Sozialversicherungsträger, bei denen Migranten unabhängig von ihrer jeweiligen Staatsangehörigkeit gleichberechtigt mit Deutschen teilnehmen können.

11 Vgl. Bertelsmann Stiftung (Hrsg.), Demokratie und Integration in Deutschland. Politische Führung und Partizipation aus Sicht von Menschen mit und ohne Migrationshintergrund, Gütersloh 2009. Siehe auch die Ergebnisse einer repräsentativen Umfrage von Bernhard Kornelius / Dieter Roth, in: Bertelsmann Stiftung (Hrsg.), Politische Partizipation in Deutschland, Gütersloh 2004, S. 27, bei denen immerhin das Kommunalwahlrecht für EU-Bürger als Beispiel für die in den letzten Jahren erweiterte Formen im Spektrum politischer Partizipation genannt werden. 
schen Kinderschutzbund und Terre des hommes. Im September 1987 erklärte auch der Deutsche Paritätische Wohlfahrtsverband, dass die „Einführung des kommunalen Wahlrechts für Ausländer (...) ein notwendiger Schritt zur weiteren Gleichberechtigung" sei. Die gleiche Forderung hatten bereits 1986 auch der Deutsche Gewerkschaftsbund (DGB), speziell die IG Metall, erhoben. Die Europa-Union Deutschlands bekräftigte in ihrer Entschließung von 1986 den bereits 1971 von ihrem Bundeskongress gefassten Beschluss, den Ausländern mit unbefristetem Aufenthaltsrecht das aktive und passive Wahlrecht in der kommunalen, beruflichen und sozialen Selbstverwaltung sowie zu den betrieblichen Arbeitnehmervertretungen zu verleihen. Die Evangelische Kirche in Deutschland (EKD) betonte in ihrer Stellungnahme von 1988, dass auf dem Wege der vollen Einbürgerung Zwischenstufen eingefügt werden könnten. Das kommunale Wahlrecht als Teil eines umfassenden Niederlassungsrechts sei als eine derartige Zwischenstufe anzusehen.

Die politischen Parteien erwähnten das Thema ebenfalls. Positive Äußerungen kamen 1988 vom Bundesvorstand der SPD und 1984 von den Grünen. Während die FDP das Kommunalwahlrecht nur unter der Voraussetzung der „Verbürgung der Gegenseitigkeit“ gewähren wollte, lehnte die CDU ein solches ausdrücklich ab. ${ }^{12}$ Sehr ausführlich wurde die Frage der Zulässigkeit des kommunalen Ausländerwahlrechts unter Juristen diskutiert. ${ }^{13}$

Parallel zu diesen gesellschaftspolitischen Debatten begann 1977 die Diskussion über die Einführung des Kommunalwahlrechts für Unionsbürger in den politischen Organen der EU, insbesondere im Europäischen Parlament. 1988 legte die EG-Kommission ihren, 1994 dann verabschiedeten, Richtlinienvorschlag zum Wahlrecht der Angehörigen der Mitgliedstaaten bei den Kommunalwahlen im Aufenthaltsstaat vor. ${ }^{14}$

\section{Das Urteil des Bundesverfassungsgerichts vom 31. Oktober 1990}

Das Bundesverfassungsgericht hat die Frage nach der rechtlichen Zulässigkeit des kommunalen Wahlrechts für Drittstaatsangehörige in zwei Urteilen zu Landesgesetzen in Schleswig-Holstein und Hamburg vom 31. Oktober $1990^{15}$ beantwortet und das kommunale Ausländerwahlrecht unter Hinweis auf die fehlende Zugehörigkeit der Ausländer zum deutschen Volk ${ }^{16}$ und unter Berufung auf das Homogenitätsprinzip (unter anderem übereinstimmende verfassungsrechtliche Voraussetzungen für die Wahlberechtigung auf Bundes-, Landes- und Kommunalebene) für unvereinbar mit dem Grundgesetz erklärt. In der Entscheidung zum Landesgesetz in Schleswig-Holstein kommt die damals noch herrschen-

12 Belege für die erwähnten Stellungnahmen bei Klaus Sieveking / Klaus Barwig / Klaus Lörcher / Christoph Schumacher (Hrsg.), Das Kommunalwahlrecht für Ausländer, Baden-Baden 1989, S. $269-280$.

13 Näheres Manfred Zuleeg, Juristische Streitpunkte zum Kommunalwahlrecht für Ausländer, in: Zeitschrift für Ausländerrecht und Ausländerpolitik 1988, S. 13 - 20, mit zahlreichen Verweisen auf die Vertreter der unterschiedlichen Meinungen.

14 Vgl. Kommission der europäischen Gemeinschaften, KOM (1988) 371 endgültig. Nachweise zur damaligen Diskussion in der EG bei Klaus Sieveking / Klaus Barwig / Klaus Lörcher / Christoph Schumacher (Hrsg.), a.a.O. (Fn. 12), S. $281-326$.

15 Vgl. BVerfGE 83, S. 37 (Schleswig-Holstein) und BVerfGE 83, S. 60 (Hamburg).

16 Vgl. Art. 20 Absatz 2 GG lautet allerdings: „Alle Staatsgewalt geht vom Volke aus.“ 
de Ansicht einer von ethnischen Kriterien bestimmten Vorstellung des deutschen Staatsvolks zum Ausdruck: Das Volk, von dem die Staatsgewalt ausgeht, ist das deutsche Volk, das ausschließlich von den deutschen Staatsangehörigen (und ihnen nach Art. 116 Abs. 1 GG gleichgestellten Personen) gebildet wird. Für Unionsbürger hat das Gericht ein Kommunalwahlrecht allerdings nicht ausgeschlossen („Öffnungsklausel“), dagegen auf das Staatsangehörigkeitsrecht und die dort vom (einfachen) Gesetzgeber auszugestaltende Möglichkeit der Einbürgerung von Ausländern verwiesen. ${ }^{17}$

Im Rahmen der Ratifikation des Maastrichter Vertrags über die Europäische Union wurde 1992 in Deutschland das Kommunalwahlrecht für Unionsbürger ${ }^{18}$ durch ein das Grundgesetz änderndes Gesetz ermöglicht. ${ }^{19}$

\section{Die Einbürgerungsfrage als neuer Bezugspunkt der Debatte über die Erweiterung politischer Beteiligung}

Anstelle der Einräumung des Kommunalwahlrechts für Drittstaatsangehörige hatte das BVerfG auf den Weg der erleichterten Einbürgerung im Rahmen des Staatsangehörigkeitsrechts verwiesen. Damit könne die angestrebte Gleichstellung, insbesondere die Wahlberechtigung auf allen politischen Ebenen erreicht werden. ${ }^{20}$ Die vorangegangenen gesellschaftlichen Debatten erhielten damit einen deutlichen Dämpfer. So wurde bei Bemühungen um eine Verbesserung der politischen Beteiligung von Drittstaatlern auf das Schleswig-Holstein-Urteil und die dort erwähnte erforderliche und mögliche Veränderung des Einbürgerungsrechts verwiesen. Damit wurde die Frage nach politischen Beteiligungsrechten der Migranten faktisch auf einen „staatsangehörigkeitsrechtlichen Einbürgerungszwang" reduziert. In der Migrationsforschung wird dies als Assimilierungsdruck bezeichnet.

In Folge dieser gesellschaftspolitischen Einschätzung wurde in den 1990er Jahren nach langwierigen Debatten das Staatsangehörigkeitsrecht geändert. ${ }^{21} 1990$ wurden staatsangehörigkeitsrechtliche Sonderregelungen in das damalige neue Ausländergesetz aufgenom-

17 Die Einbürgerung wurde inzwischen erleichtert, siehe das Gesetz zur Reform des Staatsangehörigkeitsgesetzes vom 15. Juli 1999 (BGBl. I, S. 1618), zuletzt geändert durch Artikel 5 des Gesetz zur Umsetzung aufenthalts- und asylrechtlicher Richtlinien der Europäischen Union vom 19. August 2007 (BGBl. I, S. 1970).

18 Vgl. Richtlinie 94/80/EG, ABl. EG Nr. L 368, S. 38.

19 Ergänzung von Satz 3 in Art. 28 Abs. 1 GG durch das Gesetz vom 21. Dezember 1992 (BGBl. I, S. 2086). In Erfüllung dieser Grundgesetzänderung sind in den Wahlgesetzen der Bundesländer Wahlvorschriften zum Wahlrecht und der Wählbarkeit der Unionsbürger auf kommunaler Ebene erlassen worden. Die verfassungsrechtliche Durchbrechung des Prinzips der deutschen Staatsangehörigkeit als Voraussetzung der Wahlberechtigung bei Kommunalwahlen durch den Maastrichter Vertrag von 1993 wird mit der „Öffnungsklausel“ (obiter dictum) der Entscheidung des BVerfG gerechtfertigt, ohne dass das Gericht selbst oder die Wissenschaft bis heute die widersprüchliche Aussage aufgelöst hätten, dass Bürger ohne deutsche Staatsangehörigkeit (Unionsbürger) ein kommunales Wahlrecht innehaben.

20 Dementsprechend wurde das Staatsangehörigkeitsrecht neu gefasst. Vgl. Gesetz zur Reform des Staatsangehörigkeitsgesetzes vom 15. Juli 1999, a.a.O. (Fn. 17).

$21 \mathrm{Zu}$ Fragen des Staatsangehörigkeitsrechts siehe die Kommentierung des Gesetzes, in: Rainer M. Hofmann / Holger Hoffmann (Hrsg.), Ausländerrecht. Handkommentar, Baden-Baden 2008, S. 1983. 
men, mit denen erstmals Rechtsansprüche auf Einbürgerung eingeräumt wurden. Diese neuen Regelungen wurden mit dem Zuwanderungsgesetz von 1994 in das Staatsangehörigkeitsrecht überführt. Die jüngsten Änderungen im Einbürgerungsrecht erfolgten durch das so genannte Richtlinienumsetzungsgesetz von 2007.22 Damit wurden Anregungen der Ständigen Konferenz der Innenminister zum Einbürgerungsverfahren aufgegriffen, mit denen bundesweit gleiche Standards der Einbürgerung gelten sollen. Zu den Neuerungen gehört auch der Nachweis mündlicher und schriftlicher Deutschkenntnisse auf einheitlichem Niveau, weiterhin höhere Anforderungen an die Rechtstreue der Einbürgerungsbewerber, die Einführung von Einbürgerungskursen, in denen staatsbürgerliches Wissen sowie Grundsätze und Werte der Verfassung vermittelt werden, und die Einführung von Nachweisen für eine erfolgreiche Teilnahme an diesen Kursen. ${ }^{23}$

Eine bedeutende Änderung des Staatsangehörigkeitsrechts erfolgte durch die teilweise Einführung des Erwerbs der Staatsangehörigkeit durch Geburt von Kindern der Migranten in Deutschland. Damit erfuhr die ethnische Einengung des Volksbegriffs durch das Bundesverfassungsgericht eine weitere Durchbrechung: Zum einen wurde das prinzipielle Verbot der Mehrstaatsangehörigkeit gelockert und damit eine hohe Hürde bei der Einbürgerung von Ausländern abgebaut (Entstehen einer neuen Kategorie von Migranten: „Deutsche mit Migrationshintergrund“); zum anderen wurde 2000 ausschließlich für Neugeborene aus Migrantenfamilien der Erwerb der deutschen Staatsangehörigkeit auf Grund der Geburt in Deutschland nach dem Territorialitätsprinzip (,ius soli“, vgl. $\$ 4$ Abs. 3 StAG im Rahmen des so genannten „Optionsmodells“) anstelle des sonst im deutschen Recht geltenden Abstammungsprinzips (,ius sanguinis“) ermöglicht.

\section{Einbürgerung als Weg zur politischen Beteiligung der Migranten?}

Neuere Untersuchungen über die Einbürgerung von Ausländern in Deutschland zeigen, dass zwischen dem Einbürgerungspotential (Ausländer, die die Voraussetzungen der Einbürgerung erfüllen) und der Zahl der tatsächlichen Einbürgerungen eine beträchtliche Diskrepanz besteht. ${ }^{24}$ Danach betrug die Einbürgerungsquote 2007 lediglich 1,67 Prozent der 4,1 Millionen der im Ausländerzentralregister erfassten Personen ab 16 Jahren, die die grundsätzlich erforderliche Mindestaufenthaltsdauer von acht Jahren für eine Anspruchseinbürgerung nach $\$ 10$ Abs. 1 StAG aufweisen. ${ }^{25}$

22 Vgl. Gesetz zur Reform des Staatsangehörigkeitsgesetzes vom 15. Juli 1999, a.a.O. (Fn. 17). Darin wurden aufenthalts- und asylrechtliche Richtlinien der EU umgesetzt.

23 Weitere Einzelheiten hierzu enthält der 7. Bericht über die Lage der Ausländerinnen und Ausländer in Deutschland, a.a.O. (Fn. 8), S. 139 ff. Die Umsetzung der neu gefundenen Regelungen wird laufend evaluiert.

24 Vgl. die sehr differenzierte Analyse mit zahlreichen statistischen Angaben und Erläuterungen auch zu der historischen Entwicklung sowie rechtsvergleichenden Hinweisen des Einbürgerungsrechts von Dietrich Thränhardt, Einbürgerung. Rahmenbedingungen, Motive und Perspektiven des Erwerbs der deutschen Staatsangehörigkeit, Gutachten der Friedrich-Ebert-Stiftung, Berlin 2008; Susanne Worbs, Die Einbürgerung von Ausländern in Deutschland, Working Paper 17 der Forschungsgruppe des Bundesamtes für Migration und Flüchtlinge, Nürnberg 2008, S. 21, S. 28 ff.

25 Insgesamt wurden im Jahre 200796.395 Drittstaatsangehörige eingebürgert (vgl. Statistisches Bundesamt, Bevölkerung und Erwerbstätigkeit. Einbürgerungen 2007, Fachserie 1, Reihe 2.1, S. 26 ff). Die durchschnittliche Aufenthaltsdauer der 4.407.645 im gleichen Jahr in Deutschland 
Angesichts des im deutschen Staatsangehörigkeitsrecht (mit Ausnahmen) geltenden Grundsatzes der Vermeidung von Mehrstaatigkeit und der neuerdings diskutierten Einbürgerungstests erweist sich der Weg des Erwerbs der deutschen Staatsangehörigkeit offenbar als eine Sackgasse, erhöhte politische Beteiligung der Migranten und deren Gleichberechtigung mit Unionsbürgern zu erreichen. Solange die Einbürgerung der einzige Weg zur vollen rechtlichen Gleichstellung sein soll, müssten die Hindernisse abgebaut werden. Das bezieht sich zum Beispiel auch auf den Grundsatz der Vermeidung von Mehrstaatigkeit. Diese wird zwar mittlerweile in etwa 50 Prozent der Einbürgerungen hingenommen, der Grundsatz hat sich aber inzwischen zur Ausnahme verkehrt. Zum Beispiel werden je nach Bereitschaft der ausländischen Staaten zur Entlassung ihrer Bürger aus der heimatlichen Staatsangehörigkeit Einbürgerungen mit beziehungsweise ohne Anerkennung der Mehrstaatigkeit ausgesprochen. Bei türkischen Staatsangehörigen erfolgen Einbürgerungen in der Regel ohne Hinnahme von Mehrstaatigkeit, bei anderen Staaten, bei denen nachgewiesene Schwierigkeiten mit der Entlassung aus der Staatsangehörigkeit bestehen, wird Mehrstaatigkeit hingenommen.

Anders liegen die Probleme bei hier geborenen Kindern ausländischer Eltern. Hier gilt das so genannte Optionsmodell26: Wenn die seit 2000 geborenen Kinder ausländischer Eltern 18 Jahre alt sind, müssen sie sich entweder für die deutsche oder die über ihre Eltern erworbene ausländische Staatsbürgerschaft entscheiden. ${ }^{27}$ Für die seit 2000 geborenen Doppelstaatsbürger beginnt damit die Optionspflicht im Jahr 2018. Diese wird aus integrationspolitischen Gründen nicht für sinnvoll gehalten und die Aufhebung des Optionsmodells politisch debattiert. ${ }^{28}$

Die Gründe für die großen Diskrepanzen bei der Umsetzung der gesetzlichen Neuerungen bei der Einbürgerung werden hauptsächlich in administrativen Hindernissen und Unzulänglichkeiten gesehen, insbesondere in der Länge der Verfahren, seiner Bindung an Rechtsakte anderer Staaten, was die Antragsteller von diesen Staaten abhängig, zahlungspflichtig und im Extrem erpressbar macht, und der Auslegung des Gesetzes in Bezug auf die Tolerierung bei der Beibehaltung der früheren Staatsangehörigkeit(en). ${ }^{29}$ Angesichts der mit den Einbürgerungsanträgen verbundenen bürokratischen sowie finanziellen Hürden und der die im Grunde einbürgerungsbereiten Personen diskriminierenden Verfahren bleibt die Beseitigung des Demokratiedefizits bei den Eingewanderten aufgegeben. Als ein möglicher Ausweg erscheint konsequenterweise die erweiterte Hinnahme der Mehrstaatigkeit. Vor

lebenden Drittstaatsangehörigen betrug 9,6 und mehr Jahre (vgl. Statistisches Bundesamt, Bevölkerung und Erwerbstätigkeit. Ausländische Bevölkerung. Ergebnisse des Ausländerzentralregisters 2007, Fachserie 1, Reihe 2). Nach einer Presseerklärung der Integrationsbeauftragten vom 2. September 2008 wenden zu viele Einbürgerungsbehörden die neue Regelung des $\$ 10$ Abs. 3 StAG nicht an, wonach die Mindestaufenthaltszeiten in Deutschland auf sechs Jahre verkürzt werden können, wenn besondere Integrationserfolge, etwa sehr gute deutsche Sprachkenntnisse, dokumentiert sind.

$26 \mathrm{Zu}$ den Inkonsistenzen des Optionsmodells bei Jugendlichen aus EU-Staaten siehe Dietrich Thränhardt, a.a.O. (Fn. 24), S. 42.

27 Vgl. \$4, 29 StAG.

28 Vgl. Entwurf der eines Gesetzes zur Änderung des Staatsangehörigkeitsrechts der SPD-Fraktion vom 26. Februar 2010, BT-Drs. 17/773. Auch die Länder Berlin und Bremen wollen die Optionspflicht aufheben, vgl. BR-Drs. 142/10.

29 Siehe im Einzelnen bei Dietrich Thränhardt, a.a.O. (Fn. 24), S. 21 ff. 
diesem Hintergrund sollen die Möglichkeiten politischer Partizipation wenigstens auf kommunaler Ebene erweitert werden: Eine Gleichstellung der meist schon über zehn Jahre ansässigen Drittstaatenangehörigen herzustellen entspricht rechts- und integrationspolitischer Vernunft, ohne dass grundsätzliche Verfassungsnormen verletzt würden. Die Kommunalparlamente sind die Foren, auf denen Konflikte des Alltagslebens in demokratisch organisierter und legitimierter Form ausgetragen werden. An diesen Orten wird deshalb mit darüber entschieden, wie demokratisch, konfliktfähig und lebenswert unsere Gesellschaft wirklich ist. Deshalb sollten sich alle unsere benachbarten Einwohner wenigstens an Wahlen zu den Vertretungskörperschaften von Städten und Gemeinden beteiligen dürfen.

\section{Politische Initiativen in Deutschland seit 1990}

Ungeachtet der verfassungsrechtlichen Lage und der damit einhergehenden Fokussierung rechtspolitischer Debatten auf die Einbürgerungsfrage gab das Land Hessen 1997 mit seiner Bundesratsinitiative zur Änderung des Grundgesetzes einen Anstoß für neuere Überlegungen zur Einführung eines kommunalen Ausländerwahlrechts. ${ }^{30} 1998$ kündigten SPD und Bündnis 90/Die Grünen in ihrer Koalitionsvereinbarung an, sich für das allgemeine kommunale Ausländerwahlrecht für dauerhaft im Bundesgebiet lebende Migranten einzusetzen. Verwirklicht wurde dies nicht. Nach dem Koalitionsvertrag von CDU, CSU und SPD vom 11. November $2005^{31}$ sollte die Frage des kommunalen Wahlrechts für Ausländer, die keine EU-Bürger sind, geprüft werden.

Im März $2007^{32}$ erklärte die Bundesregierung in der Antwort auf die Kleine Anfrage der Fraktion Die Linke, dass nach dem Urteil des Bundesverfassungsgerichts von 1990 die Einführung des kommunalen Wahlrechts für Drittstaatenangehörige verfassungsrechtlich zulässig sei. ${ }^{33}$ Es sei für sie jedenfalls kein Anlass für gesetzgeberische Entscheidungen, dass in den EU-Mitgliedstaaten Belgien, Dänemark, Finnland, Großbritannien, Irland, Niederlande, Portugal, Spanien, Estland, Litauen, Slowakei, Slowenien, Ungarn und Tschechische Republik sowie Island ein Wahlrecht auf kommunaler Ebene für Drittstaatenangehörige unter unterschiedlichen gesetzlichen Voraussetzungen bestehe. ${ }^{34}$ Die dafür verfassungsrechtlich erforderliche qualifizierte Bundestagsmehrheit sei nicht absehbar. In dem Aus-

30 Zur Entwicklung neuer Debatten siehe ausführlich Klaus Sieveking, Kommunalwahlrecht für Drittstaatsangehörige - „kosmopolitische Phantasterei“ oder Integrationsrecht für Einwanderer?, in: Zeitschrift für Ausländerrecht und Ausländerpolitik 2008, S. 121 - 126.

$31 \mathrm{Vgl}$. Zeitschrift für Ausländerrecht und Ausländerpolitik 2005, S. 385.

32 Vgl. BT-Drs. 16/4666 vom 13. Februar 2007. Siehe auch BT-Drs. 16/4361 zur „Umsetzung des Prüfauftrages zur Einführung des kommunalen Wahlrechts für Drittstaatenangehörige" vom 21. Februar 2007.

33 Nach Artikel 79 Abs. 3 GG sei das nicht grundsätzlich ausgeschlossen, da das Gericht die Einführung eines kommunalen Wahlrechts für Unionsbürger ausdrücklich mit Artikel 79 Abs. 3 GG für vereinbar erklärt habe. Dies stelle allerdings eine auf europäischem Recht beruhende zulässige Privilegierung dar, die dem Integrationsprozess innerhalb der Europäischen Union Rechnung trage.

34 Unter Berufung auf eine Studie der Österreichischen Stiftung für Politikberatung und Politikentwicklung vom Februar 2007; vgl. Werner T. Bauer, Das kommunale Ausländerwahlrecht im europäischen Vergleich, Wien 2007. 
schluss vom Kommunalwahlrecht sehe die Bundesregierung weder eine rechtliche Benachteiligung von Nicht-EU-Bürgern noch ein demokratisches Defizit. Ebenso wenig sei eine Verfassungsänderung deshalb geboten, weil die durchschnittliche Aufenthaltsdauer aller Drittstaatenangehörigen 16,8 Jahre betrage und 21 Prozent in Deutschland geboren seien. Das Grundgesetz müsse diesem Wandel der tatsächlichen Verhältnisse nicht Rechnung tragen und den Begriff des Staatsvolkes nicht entsprechend weiter fassen.

Anfang Juli 2007 stellte die Fraktion Die Linke im Bundestag den Antrag, ein kommunales Wahlrecht für Drittstaatenangehörige einzuführen. ${ }^{35}$ Im September 2007 beantragte die Regierung des Landes Rheinland-Pfalz im Bundesrat die erneute Einbringung des bereits 1997 vorgelegten Entwurfs eines Gesetzes zur Änderung des Grundgesetzes. Dieser Antrag wurde abgelehnt.

Am 10. Oktober 2007 legte die Fraktion Bündnis 90/Die Grünen im Bundestag den „Entwurf eines Gesetzes zur Änderung des Grundgesetzes (Kommunales Ausländerwahlrecht)“ vor. Damit sollte - unter Hinweis auf die Diskussion in der Gemeinsamen Verfassungskommission von Bundestag und Bundesrat aus dem Jahre $1993^{36}$ - die Integration aller in Deutschland wohnenden ausländischen Mitbürger durch Einräumung des Kommunalwahlrechtes gefördert werden, um die Ungleichbehandlung zwischen Unionsbürgern und den übrigen Ausländern zu beseitigen. In einem Interview hielt der Staatsrechtler Josef Isensee den Vorstoß von Linken und Grünen für „schlicht verfassungswidrig “37. Durch „kosmopolitische Phantastereien“ werde der Versuch unternommen, das Staatsvolk zu überrollen und eine „demokratiewidrige Fremdbestimmung“ einzuführen. Die kommunalen Gebietskörperschaften seien „Teil der Staatsgewalt und bedürfen demokratischer Legitimation. Diese kann nur vom ,demos', dem Volk, das durch die Staatsangehörigkeit definiert werde, erteilt werden“. Diese Regelung sei dadurch „etwas modifiziert“ worden, dass auch die Unionsbürgerschaft das Wahlrecht ermögliche. Der Bericht zu diesem Interview zitiert demgegenüber den Staatsrechtslehrer Hans-Peter Schneider. Dieser sieht in der Tatsache, dass EU-Bürger an Kommunalwahlen in Deutschland teilnehmen können, ein Argument dafür, dass die deutsche Staatsangehörigkeit nicht mehr alleinige Voraussetzung für das Wahlrecht sein kann. Deutschland sei heute ein Einwanderungsland. „Menschen, die jahrelang hier leben, sind keine Ausländer mehr, sondern Inländer. Sie müssen auch mitbestimmen dürfen, wenn es um ihre Angelegenheiten geht. Diese örtlichen Angelegenheiten haben mit der Staatsangehörigkeit nichts zu tun, das sind Sachthemen, die alle angehen müssen." Zudem knüpfe das Kommunalrecht an den Begriff der Einwohner und nicht an den der Staatsbürger an. ${ }^{38}$

Als Antwort auf die Frage, wer das Volk als demokratisches Legitimationssubjekt ist ${ }^{39}$, wird ungeachtet des inzwischen eingetretenen Verfassungswandels nach wie vor auf das Urteil des Bundesverfassungsgerichts von 1990 verwiesen. Seine Vorstellung von der Bedeutung des Volksbegriffs durch Gleichsetzung des Demokratieprinzips des Art. 20 Abs. 2

35 Vgl. BT-Drs. 16/5904 vom 4. Juli 2007.

36 Vgl. BR-Drs. 800/93 vom 5. November 1993, S. 97 ff.

37 Vgl. Susanne Kailitz, Ein Kreuzchen in der Wahl-Heimat, in: Das Parlament vom 29. Oktober/5. November 2007, S. 6.

38 Sämtliche Zitate ebenda.

39 Hierzu und zu den folgenden Überlegungen Astrid Wallrabenstein, Das Verfassungsrecht der Staatsangehörigkeit, Baden-Baden 1999, S. 102 ff. mit zahlreichen Nachweisen. 
GG mit dem Staatsvolk der Staatsangehörigen als deutschem Volk steht der inzwischen wohl von den meisten Staatsrechtslehrern vertretenen Ansicht von der sprachlichen und normativen Offenheit des Volksbegriffs entgegen.

\section{Neuere Positionen von Parteien und gesellschaftlichen Organisationen}

Die 27. Ordentliche Bundesdelegiertenkonferenz der Bündnisgrünen nahm im November 2007 in Nürnberg den Antrag zur „Erweiterung des Kommunalwahlrechts auf die NichtEU-Bürgerinnen und Bürger“ an. In ihrem Wahlprogramm 2009 forderten sie, dass auch Nicht-EU-Bürger ein kommunales Wahlrecht erhalten sollen. ${ }^{40}$ Von Seiten der SPD gibt es soweit ersichtlich keine neuen programmatischen Aussagen zum Thema. Nach einer Bekundung des Vorsitzenden des Innenausschusses im Bundestag aus dem Jahre 2009 wollte die SPD-Fraktion gegenüber ihrem Koalitionspartner CDU/CSU dafür werben, das kommunale Wahlrecht für langjährig in Deutschland lebende Ausländer einzuführen ${ }^{41}$ - offenbar ohne Erfolg. Die Wahlaussage der CDU 2009 beschränkte sich auf die Feststellung, ein gutes Zusammenleben von Einheimischen und Migranten ermöglichen zu wollen. ${ }^{42}$

Eine ausführliche Debatte erfolgte im Rahmen einer Öffentlichen Anhörung des Innenausschusses des Bundestages im September 2008, zu der Sachverständige schriftliche und mündliche Stellungnahmen zu den genannten Entwürfen der Bündnisgrünen und der Linken vorlegten. ${ }^{43}$

Mit der Konstituierung des 17. Bundestags nach der Wahl am 27. September 2009 mussten parlamentarische Aktivitäten neu eingebracht werden. ${ }^{44} \mathrm{Als}$ erste legte die Fraktion der SPD unter erneuter Bezugnahme auf die Diskussion in der Gemeinsamen Verfassungskommission von Bundestag und Bundesrat ${ }^{45}$ den „Entwurf eines ... Gesetzes zur Änderung des Grundgesetzes (Art. 28 Absatz 1)“ vor. ${ }^{46}$ Die Linke stellte erneut einen Antrag „Kommunales Wahlrecht für Drittstaatsangehörige einführen“ “77, während die Bündnisgrünen ihren vom Bundesrat am 26. September 2007, aber vom Bundestag wegen Ablaufs der Wahlperiode nicht mehr beschlossenen Gesetzentwurf entsprechend neu eingebrachte. ${ }^{48}$ Alle drei Initiativen betonen die Notwendigkeit, die Integration der hier wohnenden aus-

40 Vgl. Bündnis 90/Die Grünen, Bundestagswahlprogramm 2009, S. 164.

41 Siehe dazu den Kommentar von Sebastian Edathy, Kommunales Wahlrecht für Drittstaatler, in: Forum Migration 2007, H. 10, S. 4.

42 Kurzversion des Regierungsprogramms 2009-2013 von CDU und CSU, Berlin 2009. Siehe auch Christoph Hauschild, Die integrationspolitischen Ziele im Koalitionsvertrag, in: Zeitschrift für Ausländerrecht und Ausländerpolitik 2010, S. $161 \mathrm{ff}$.

43 Das Anhörungsprotokoll und die Stellungnahmen (auch die des Verfassers) sind einzusehen unter http://www.bundestag.de/bundestag/ausschuesse/a04/anhoerungen/Anhoerung_16/Protokoll. pdf (Abruf am 10. August 2010) und http://www.bundestag.de/bundestag/ausschuesse/a04/anhoerungen/Anhoerung_16/Stellungnahmen_SV/index.html (Abruf am 10. August 2010).

44 Die Vorlagen im Deutschen Bundestag gelten am Ende der Wahlperiode als erledigt (Grundsatz der Diskontinuität), vgl. Art. 39 GG und $\$ 125$ GOBT.

45 Vgl. BT-Drs. 12/6000 vom 5. November 1993.

46 Vgl. BT-Drs. 17/1047 vom 16. März 2010.

47 Vgl. BT-Drs. 17/1146 vom 23. März 2010.

48 Vgl. BT-Drs. 17/1150 vom 23. März 2010. 
ländischen Mitbürger zu fördern sowie die Ungleichbehandlung von Unionsbürgern und den übrigen Ausländern zu beseitigen. Es bleibt abzuwarten, welche parlamentarischen Folgen diese jüngsten Vorstöße haben werden. ${ }^{49}$

Auch von zivilgesellschaftlichen Gruppen gibt es neuere Stellungnahmen. So hat Pro Asyl im Rahmen einer Kampagne gegen Rassismus und Diskriminierung der Bundesregierung vorgeworfen, dass trotz Integrationsgipfeln und Integrationsplänen Anstrengungen und Maßnahmen zur Überwindung gesellschaftlicher Ausgrenzung und Eröffnung von Teilhabechancen unterblieben. Besonders die langjährige Forderung nach einem kommunalen Wahlrecht für Drittstaatler bliebe in Deutschland unerfüllt, obwohl es in vielen anderen EU-Staaten bereits erfolgreich praktiziert werde. ${ }^{50}$ Pro Asyl, der Interkulturelle Rat in Deutschland und der DGB haben zum Wahljahr 2009 eine Gemeinsame Erklärung zu „Prioritäten für die deutsche und europäische Migrations- und Flüchtlingspolitik“ veröffentlicht. In diesem Positionspapier wird ein kommunales Wahlrecht für alle rechtmäßig und dauerhaft in Deutschland lebenden Bürger und eine Änderung des Art. 28 GG gefordert. $^{51}$

Die EKD hat zuletzt 2002 in einer Handreichung das Thema aufgegriffen. ${ }^{52}$ Die Landesarbeitsgemeinschaft der kommunalen Migrantenvertretungen Nordrhein-Westfalen, eine Gruppe von kommunalen Aktionsgruppen und den Spitzenverbänden der sieben Wohlfahrtsverbände in Deutschland, führt seit 2008 wiederholt Pressekampagnen mit der Forderung nach Einführung des kommunalen Wahlrechts für Migranten durch. Derzeit läuft eine Internetkampagne zur Sammlung von Unterschriften. ${ }^{53}$

\section{Neuere Entwicklungen in der EU}

Eine wichtige Unterstützung hat die Forderung nach politischer Beteiligung der Migranten durch europäische Entwicklungen der letzten Jahre erfahren. ${ }^{54}$ Die Einführung des kom-

49 Siehe dazu die Diskussion in der 34. Sitzung des Bundestags am 25. März 2010.

50 Vgl. PRO ASYL, Presseerklärung vom 21. März 2008, http://www.proasyl.de/de/presse/presseuebersicht/?0 (Abruf am 10. August 2010).

51 Das Positionspapier kann herunter geladen werden unter http://www.proasyl.de/de/news/detail/ news/bundestagswahl_2009 (Abruf am 10. August 2010).

52 Vgl. EKD, Zusammenleben gestalten. Ein Beitrag des Rates der EKD zu Fragen der Integration und des Zusammenlebens mit Menschen anderer Herkunft, Sprache oder Religion, in: EKDTexte 2002, Nr. 76, Rdnr. 39 - 42, 40. Es heißt dort: „Nach der Einführung des Kommunalwahlrechts für Unionsbürger bedarf es einer erneuten sorgfältigen Prüfung, auf welche Weise die Teilhabe der Nichtdeutschen an der politischen Verantwortung für das Gemeinwesen gefördert und gestärkt werden kann. Da die Verleihung des vollen Wahlrechts an alle Ausländer an verfassungsrechtlichen Hürden scheitert, sollten andere Möglichkeiten der politischen Partizipation untersucht werden, die über die beratenden Kompetenzen der Ausländerbeiräte und der Ausländerbeauftragten hinausgehen."

53 Vgl. http://www.wahlrecht-fuer-migranten.de/xd/public/content/index.html?pid=518 (Abruf am 10. August 2010). Bislang haben dort 2.084 Personen die Initiative unterstützt.

54 Ausführlich hierzu Klaus Sieveking, a.a.O. (Fn. 30); Felix Hanschmann, „Die Ewigkeit dauert lange, besonders gegen Ende" - eine rechtliche (Neu-)Bewertung des kommunalen Wahlrechts für Drittstaatsangehörige, in: ZParl, 40. Jg. (2009), H. 1, S. 74 - 85. 
munalen Wahlrechts für Unionsbürger ${ }^{55}$ hat den scheinbar unauflöslichen Zusammenhang zwischen Volkssouveränität, Staatsangehörigkeit und Wahlrecht widerlegt. Für Unionsbürger besteht das Kommunalwahlrecht ungeachtet ihrer fehlenden deutschen Staatsangehörigkeit sowohl in ihrem Herkunftsstaat als auch in ihrem Wohnsitzstaat. ${ }^{56}$ Das Gemeinschaftsrecht verbietet nicht, das Kommunalwahlrecht auf Drittstaatsangehörige auszudehnen. ${ }^{57}$ Der Ausschluss der Drittstaatsangehörigen führt zu einer fortbestehenden Ungleichbehandlung von Unionsbürgern einerseits und dauerhaft in Deutschland lebenden Drittstaatsangehörigen andererseits. Dies widerspricht vor allem der allseits erwünschten Integration - ungeachtet der anhaltenden Meinungsunterschiede über die integrativen Effekte eines Ausländerwahlrechts. Festzustellen ist jedenfalls, dass eine große Zahl der EUMitgliedstaaten den in ihrem Land wohnenden Migranten eine Möglichkeit eröffnet hat, sich an Kommunalwahlen zu beteiligen. ${ }^{58}$

Weiter hat die EU 2003 für langfristig in den Mitgliedsländern lebende Drittstaatenangehörige rechtliche Konsequenzen in Bezug auf deren Aufenthaltsstatus gezogen und ihnen eine dem Unionsbürger-Status angeglichene Rechtsposition zugestanden: Mit der Richtlinie 2003/109/EG betreffend die Rechtsstellung der langfristig aufenthaltsberechtigten Drittstaatenangehörigen ${ }^{59}$ wird diesen nach fünfährigem rechtmäßigen Aufenthalt der Titel „Langfristige Aufenthaltsberechtigung - EG“ und - unter näher festgelegten Umständen - auch ein Weiterwanderungsrecht ${ }^{60}$ innerhalb der EU gewährt. Diese Richtlinie wurde 2007 durch das Gesetz zur Umsetzung aufenthalts- und asylrechtlicher Richtlinien der Europäischen Union in das deutsche Aufenthaltsgesetz umgesetzt. ${ }^{61}$ Damit ist die aufenthaltsrechtliche Gleichstellung von Drittausländern mit Daueraufenthaltsrecht und Unionsbürgern erreicht worden.

Diese Richtlinie steht - wie es in der dritten Erwägung ihres Textes heißt - in Einklang mit den Grundrechten und berücksichtigt die Grundsätze, die insbesondere durch die Europäische Konvention zum Schutze der Menschenrechte und Grundfreiheiten und die Charta der Grundrechte der Europäischen Union anerkannt wurden. Die zwölfte Erwägung der Richtlinie betont: Um ein echtes Instrument zur Integration von langfristig Aufenthaltsberechtigten in die Gesellschaft, in der sie leben, darzustellen, sollten langfristig Aufenthaltsberechtigte nach Maßgabe der in dieser Richtlinie festgelegten Bedingungen in

55 Vgl. Art. 19 Abs. 1 EG.

56 Bei der Wahl zum Europäischen Parlament ist eine derartige doppelte Stimmabgabe dagegen nicht möglich.

57 Siehe dazu die Urteile des EuGH vom 12. September 2006 in der Rechtsache C-145/04 - Spanien / Vereinigtes Königreich, ABl. C. 281 vom 18. November 2006, 2, EuGHE I 2006, 7917 und vom 12. September 2006 in der Rechtsache C-300/04 - Mike Eman / Benny Sevinger, ABl. C. 281 vom 18. November 2006, 5, EuGHE I 2006, 8055.

58 Vgl. Werner T. Bauer, a.a.O. (Fn. 34); Klaus Sieveking / Klaus Barwig / Klaus Lörcher / Christoph. Schumacher (Hrsg.), a.a.O. (Fn. 12), S. 330, S. 385.

$59 \mathrm{Vgl}$. ABl. EG 2004, Nr. L 16/44.

60 Die Wahrnehmung dieses Rechts hätte zum Beispiel für Drittausländer in den Niederlanden bei Weiterwanderung nach Deutschland die Konsequenz des Verlusts des Kommunalwahlrechts.

61 Gesetz über den Aufenthalt, die Erwerbstätigkeit und die Integration von Ausländern im Bundesgebiet (Aufenthaltsgesetz - AufenthG) vom 30. Juli 2004 (BGBl. I, S. 1950), zuletzt geändert durch Artikel 1 des (Richtlinienumsetzungs-)Gesetzes vom 19. August 2007 (BGBl. I, S. 1970), vgl. \$\$ 9a ff., 38, 91c AufenthG. 
vielen wirtschaftlichen und sozialen Bereichen wie die Bürger des Mitgliedstaats behandelt werden. ${ }^{62}$

\section{Aktuelle Initiativen im Spiegel völkerrechtlicher Diskussionen}

Die völkerrechtliche Entwicklung der letzten Jahre beeinflusst die Erweiterung der politischen Beteiligung der Migranten in hohem Maße: Mit der Herausbildung transnationaler Zugehörigkeiten hat die Rolle der national begrenzten Staatsangehörigkeit an Überzeugungskraft eingebüßt. Der nationale staatsbürgerrechtliche Status hat an Bedeutung verloren. Migranten sind in Zugehörigkeitsverhältnisse jenseits des Nationalstaates eingebunden. Diese Entwicklung lässt sich auch am internationalen Menschenrechtsschutz verfolgen. Mit der europa- und völkerrechtlichen Entwicklung von einklagbaren Schutzrechten bilden sich transnationale Bürgerschaftsverhältnisse heraus. Rechtspositionen, die herkömmlich ausschließlich der staatsangehörigen nationalen Bevölkerung vorbehalten waren, verselbständigen sich und werden folglich jedem Menschen unabhängig von seiner Staatsangehörigkeit zugänglich. ${ }^{63}$ Ein evidentes Beispiel hierfür ist die oben erwähnte Daueraufenthaltsrichtlinie der EG. Mit der völkerrechtlichen Universalisierung eines menschen- und sozialrechtlichen Schutzes unabhängig von der Staatsangehörigkeit der Betroffenen entwickelt sich eine "Staatsbürgerschaft des Aufenthaltsortes“, die auf multiplen Bindungen zur lokalen und regionalen Ebene sowie zu transnationalen Institutionen beruht. ${ }^{64}$ Als normative Anknüpfungspunkte für die hier vertretene Gleichbehandlung der Migranten in Bezug auf ihre politische Beteiligung an Kommunalwahlen an ihrem Aufenthaltsort sind Art. 3 Abs. 1 des GG einerseits und die Art. 14 und 16 der Europäischen Menschenrechtskonvention zu nennen.

62 Bei den erwähnten Erwägungen handelt es sich um die Erwägungen (3) beziehungsweise (12) der Richtlinie 2003/109/EG des Rates betreffend die Rechtstellung der langfristig aufenthaltsberechtigten Drittstaatsangehörigen (vgl. Fn. 59). Von der politischen Integration ist in Erwägung (12) nicht die Rede, weil die EG insoweit keine Regelungskompetenz hat.

63 Ausführlich Nikolaos Kotalakidis, Von der nationalen Staatsangehörigkeit zur Unionsbürgerschaft, Baden-Baden 2000, S. 86 f.; siehe auch Ece Ozlem Atikcan, Citizenship or Denizenship: The Treatment of Third Country Nationals in the European Union, SEI Working Paper, Nr. 85, Sussex 2006. Das Konzept der „Denizenship“ reflektiert einen neuen Rechtsstatus jenseits der traditionellen Staatsangehörigkeit. Siehe auch Karsten Nowrot, Den „Kinderschuhen“ entwachsen: Die (Wieder-)Entdeckung der rechtssoziologischen Perspektive in der Dogmatik der Völkerrechtssubjektivität, in: Zeitschrift für Rechtssoziologie, 28. Jg. (2007), H. 1, S. $21-48$.

64 Vgl. Seyla Benhabib, Die Dämmerung der Souveränität oder das Aufstreben kosmopolitischer Normen? Eine Neubewertung von Staatsbürgerschaft in Zeiten des Umbruchs, in: Regina Kreide I Andreas Niederberger (Hrsg.), Transnationale Verrechtlichung, Frankfurt am Main 2008, S. 209 - 239, S. 214, S. 230. Zur Frage von pluralen Zugehörigkeiten in sozialen Kontexten siehe Amartya Sen, Die Identitätsfalle. Warum es keinen Krieg der Kulturen gibt, München 2007, S. $38 \mathrm{ff}$. 


\section{Kommunalwahlrecht für Migranten: unverzichtbar und unaufhaltsam}

Die politischen und gesellschaftlichen Ansätze, die politische Beteiligung von Migranten ohne deutsche Staatsangehörigkeit zu verbessern, haben bislang keinen Erfolg gehabt. Das liegt vor allem daran, dass keine ausreichenden politischen Mehrheiten für eine notwendige Änderung des Grundgesetzes mobilisiert werden konnten. Offenbar sind auch die im übrigen Europa, vor allem in den Niederlanden gemachten Erfahrungen zu wenig bekannt. ${ }^{65}$ Ebenso wenig hat man Konsequenzen aus dem tatsächlich eingetretenen Verfassungswandel ziehen wollen - der Respekt vor dem Urteil des Bundesverfassungsgerichts wirkt andauernd, obwohl europa- und völkerrechtliche Entwicklungen schon länger die innere Widersprüchlichkeit des Urteils offengelegt haben. Die gesellschaftliche, insbesondere aus europawie völkerrechtlichen Gründen und Integrationserfordernissen notwendige Gleichstellung von Migranten ohne deutsche Staatsangehörigkeit, also die Gleichbehandlung der auf Dauer eingewanderten Migranten mit den Unionsbürgern, müsste als wichtigstes Ziel der deutschen Migrations- und Integrationspolitik anerkannt werden. Gerade beim Kommunalwahlrecht bewirkt derzeit die positive Statuserweiterung der Unionsbürger im Aufenthaltsstaat gegenüber Drittstaatsangehörigen eine Ungleichbehandlung. Der Ausschluss der letzteren von politischen Teilhaberechten führt zu einer Betonung ihrer politischen Desintegration, die nach den Äußerungen in allen politischen Programmen ausdrücklich nicht gewollt, aber doch faktisch vorhanden ist. Um die Integration tatsächlich zu erreichen, ist es erforderlich, dass die erwähnten bürokratischen Einbürgerungshindernisse gesenkt werden und vor allem, dass sich das politische Bewusstsein verändert, und die Ängste der Herkunftsdeutschen abgebaut werden. ${ }^{66}$

$\mathrm{Ob}$ mit der Erweiterung der politischen Beteiligung auf kommunaler Ebene das sichtbar gewordene parallele Siedlungsrecht unterschiedlicher Ethnien hin zu einem gemeinsamen Staatsvolk beeinflusst werden kann, wird zu untersuchen sein. Die Debatten über einen einheitlichen Siedlungs- und Kulturraum könnten hier jedenfalls einen weiteren Anknüpfungspunkt finden. Es ist zu erwarten, dass die Generation der heutigen Jugendlichen zu gegebener Zeit diese Debatte forcieren und mit der Einführung des Kommunalwahlrechts den notwendigen Schritt zur Gleichstellung der Migranten bei der politischen Beteiligung durchsetzen werden, zumal dann ohnehin aus Gründen der demographischen Entwicklung der größere Teil der Bevölkerung in Deutschland „Deutsche mit Migrationshintergrund“ sein werden.

65 Vgl. Kees Groenendijk, Local Voting Rights for Non-Nationals in Europe: What We Know and What We Need to Learn, in: Bertelsmann Stiftung, European Policy Centre, Migration Policy Institute (Hrsg.), Delivering Citizenship, Athen 2008, S. 49 - 72. Siehe auch Dietrich Thränhardt, a.a.O. (Fn. 24), S. $29 \mathrm{ff}$.

66 Darauf zielen bereits neuere Perspektiven der politischen Bildung ab; vgl. Dirk Lange / Ayca Polat (Hrsg.), a.a.O. (Fn. 8). 